\title{
Review Article \\ The Multiple Roles of EG-VEGF/PROK1 in Normal and Pathological Placental Angiogenesis
}

\author{
Nadia Alfaidy, ${ }^{1,2,3}$ Pascale Hoffmann, ${ }^{1,2,3,4}$ Houssine Boufettal, ${ }^{5,6}$ \\ Naima Samouh, ${ }^{5}$ Touria Aboussaouira, ${ }^{6}$ Mohamed Benharouga, ${ }^{1,2,7}$ \\ Jean-Jacques Feige, ${ }^{1,2,3}$ and Sophie Brouillet ${ }^{1,2,3,4}$ \\ ${ }^{1}$ Commissariat à l'Energie Atomique (CEA), DSV-iRTSV, 17 rue des Martyrs, 38054 Grenoble Cedex 9, France \\ ${ }^{2}$ Université Grenoble-Alpes, 38041 Grenoble, France \\ ${ }^{3}$ Institut National de la Santé et de la Recherche Médicale U1036 (INSERM U1036), Biologie du Cancer et de l'Infection, \\ Laboratoire BCI-iRTSV, CEA Grenoble, 17 rue des Martyrs, 38054 Grenoble Cedex 9, France \\ ${ }^{4}$ CHU de Grenoble, Hôpital Couple Enfant, Département de Génétique et Procréation, Centre d’Aide Médicale à la Procréation, \\ CS 10217, 38043 Grenoble Cedex 9, France \\ ${ }^{5}$ Service de Gynécologie-Obstétrique "C", Centre Hospitalier Universitaire Ibn Rochd, Faculté de Médecine et de Pharmacie, \\ Université Hassan II, Ain Chok, 1 rue des Hôpitaux-ex Banaflous, 20360 Casablanca, Morocco \\ ${ }^{6}$ Plateau Commun de Recherche, Unité de Culture Cellulaire, Faculté de Médecine et de Pharmacie, \\ 19 rue Tarek Bnou Ziad, 20360 Casablanca, Morocco \\ ${ }^{7}$ Centre National de la Recherche Scientifique, UMR 5249, 38054 Grenoble Cedex 9, France
}

Correspondence should be addressed to Sophie Brouillet; sophie_brouillet@yahoo.fr

Received 6 February 2014; Revised 8 April 2014; Accepted 14 April 2014; Published 15 May 2014

Academic Editor: Padma Murthi

Copyright (C) 2014 Nadia Alfaidy et al. This is an open access article distributed under the Creative Commons Attribution License, which permits unrestricted use, distribution, and reproduction in any medium, provided the original work is properly cited.

\begin{abstract}
Placentation is associated with several steps of vascular adaptations throughout pregnancy. These vascular changes occur both on the maternal and fetal sides, consisting of maternal uterine spiral arteries remodeling and placental vasculogenesis and angiogenesis, respectively. Placental angiogenesis is a pivotal process for efficient fetomaternal exchanges and placental development. This process is finely controlled throughout pregnancy, and it involves ubiquitous and pregnancy-specific angiogenic factors. In the last decade, endocrine gland derived vascular endothelial growth factor (EG-VEGF), also called prokineticin 1 (PROK1), has emerged as specific placental angiogenic factor that controls many aspects of normal and pathological placental angiogenesis such as recurrent pregnancy loss (RPL), gestational trophoblastic diseases (GTD), fetal growth restriction (FGR), and preeclampsia (PE). This review recapitulates EG-VEGF mediated-angiogenesis within the placenta and at the fetomaternal interface and proposes that its deregulation might contribute to the pathogenesis of several placental diseases including FGR and PE. More importantly this paper argues for EG-VEGF clinical relevance as a potential biomarker of the onset of pregnancy pathologies and discusses its potential usefulness for future therapeutic directions.
\end{abstract}

\section{Introduction}

The placenta is one of the most densely vascularized organs in the organism [1]. At term, it has developed a capillary network that is $\cong 550 \mathrm{~km}$ in length and $15 \mathrm{~m}^{2}$ in surface area $[1,2]$. During the course of 9 months, placental vascular network expansion is a dynamic process characterized by intravilli vasculogenesis followed by branching and nonbranching angiogenesis [3]. Vasculogenesis starts during the third week after conception and involves de novo formation of new vessels. This process is characterized by the formation of the first blood vessels from differentiation of pluripotent mesenchymal cells into haemangiogenic stem cells [4]. The subsequent step, angiogenesis, starts during the fifth week after conception and refers to the development of new vessels from preexisting vessels $[4,5]$. From day 32 to week 25 after conception, haemangioblastic cords formed by vasculogenesis develop into a richly branched villous capillary bed by 
two mechanisms: elongation of preexisting tubes and lateral ramification of these tubes (sprouting angiogenesis). Around week 25 , this process switches from branching to nonbranching angiogenesis $[4,5]$. Nonbranching angiogenesis occurs in mid and late gestation and it is mainly characterized by endothelial cell proliferation leading to an increase in the surface of the endothelial tissue. These processes ensure the increasing supply of gas and nutrient for the growing fetus $[4,5]$.

For many years, morphological and functional diversity among endothelia were thought to result from vascular bed-specific response to ubiquitous and tissue-restricted mediators. In this context, several ubiquitous growth factors (i.e., vascular endothelial growth factor (VEGF) and basic fibroblastic growth factor (bFGF)), as well as numerous pregnancy-specific angiogenic factors (i.e., placental growth factor (PlGF) and human chorionic gonadotropin hormone (hCG)), have been reported to regulate either the intravilli or the fetomaternal angiogenesis $[4,5]$.

The existence of tissue-specific angiogenic factors has been postulated for many years [6-9] but it only recently received confirmation when such a factor, named endocrine gland-derived vascular endothelial growth factor/ prokineticin 1 (EG-VEGF/PROK1), was finally characterized [10].

\section{EG-VEGF/PROK1 in the Placenta}

2.1. EG-VEGF, a New Angiogenic Factor Highly Expressed in the Reproductive Organs. In 2001, a novel family of angiogenic mitogens, named the prokineticins, has been characterized with restricted expression profiles and selective endothelial cell activity [10]. This family is composed of two members, EG-VEGF/PROK1 and PROK2, with multiple roles in physiological and pathological conditions. Human EGVEGF and PROK2 proteins exhibit $44 \%$ amino-acid identity and share the same G protein-coupled receptors (PROKR), termed PROKR1 and PROKR2 [11]. PROKR bind the peptide hormones EG-VEGF and PROK2, with PROK2 showing a moderately higher affinity than EG-VEGF for both receptors [11]. Although prokr1 and prokr2 genes are located on two different chromosomes (2q14 and 20p13, resp.), they encode proteins that share $85 \%$ amino acid identity and that exhibit the greatest differences in the N-terminal domains [11]. The exact functions of each receptor are not fully elucidated, but recent data reported specificities of actions in the heart and the placenta, where PROKR1 is preferentially involved in proliferation and angiogenic processes and PROKR2 is mainly implicated in endothelial permeability [12-14]. The prokineticins show different patterns of expression and preferential sites of actions. PROK2 is mainly associated with the nervous system, whereas EG-VEGF is predominantly associated with the reproductive tract and the endocrine organs, including the ovary and the placenta [10]. In the last decade, many studies have shed light on the angiogenic roles of EGVEGF in the reproductive organs. In the gonads, EG-VEGF has been reported to impact physiological and pathological angiogenic processes [15-18]. In endothelial cells isolated from steroidogenic tissues, EG-VEGF has been shown to promote proliferation, differentiation, survival, chemotaxis, and fenestration of capillary endothelial cells [10, 19, 20]. Interestingly, the effect of EG-VEGF on endothelial cells seems to be tissue specific as it has no effect on endothelial cells derived from brain capillary, aorta, umbilical vein, or cornea $[10,21]$.

\subsection{Placental Expression of EG-VEGF/PROKR Throughout} Pregnancy. EG-VEGF is the major prokineticin in the female reproductive tract. In the placenta, EG-VEGF and its receptors are highly expressed [10, 22-26]. EG-VEGF is mainly localized to the syncytiotrophoblast layer (ST) with a mild expression in the cytotrophoblast layer (CT) [22]. EG-VEGF receptor PROKR1 is abundant in the $\mathrm{CT}$, the placental microvascular endothelial cells (HPEC), and the Hofbauer cells (Ho), whereas PROKR2 is expressed by ST, HPEC, Ho, and extravillous trophoblasts (EVT) [22-25]. EG-VEGF and its receptors show a dynamic profile throughout pregnancy. In the placenta, EG-VEGF, PROKR1 and PROKR2 are predominantly expressed during the first trimester of pregnancy. During early pregnancy, EG-VEGF/PROKR1 peaks at 8-11 weeks of gestation (wg) and then gradually decreases by the end of the first trimester, whereas PROKR2 expression is maintained over the first trimester $[22,23,26]$. In nonpregnant women, circulating EG-VEGF levels are around $50 \mathrm{pg} / \mathrm{mL}$ [23]. During pregnancy, these levels significantly increase fivefold during the first trimester $(\cong 250 \mathrm{pg} / \mathrm{mL})$ and then gradually decrease to reach those observed in nonpregnant women by the end of the second trimester of pregnancy [23].

2.3. EG-VEGF/PROKR System and Placental Development. EG-VEGF is directly involved in the growth of the placental villi with multiple actions on various cell types. This factor is mainly produced by the ST $[22,23]$ and acts on the adjacent CT to increase their proliferation [27] at the expense of their differentiation to ST. This phenomenon contributes to the overall growth of the placental villi, an important aspect of placental development during the first trimester of human pregnancy. Moreover, EG-VEGF promotes the proliferation of anchoring trophoblasts and inhibits early EVT migration and invasion. In first trimester human placenta, anchoring trophoblastic plugs obstruct the spiral arteries and prevent maternal oxygenized blood from entering into the intervillous space. This physiological process creates a local hypoxic environment indispensable for normal placental and fetal development. At the end of the first trimester, anchoring trophoblast generates multilayered columns of EVT that invade the uterine blood vessels and remodel the maternal spiral arteries from minimal-flow/high-resistance vessels into larger diameter vessels with low resistance and high flow. The contribution of EG-VEGF in the formation and maintenance of the trophoblastic plugs throughout the first trimester protects the fetoplacental unit from early oxidative stress against which the first trimester placenta is not equipped $[28,29]$. Altogether, these data demonstrate that EG-VEGF is a new placental growth factor that contributes to ensure 
the maintenance of pregnancy during the first trimester of pregnancy.

\section{EG-VEGF Control of Placental Angiogenesis}

Beside its effects on the trophoblastic component of the placental villi, growing evidences established the involvement of EG-VEGF and its receptors in placental angiogenesis. The following paragraphs will discuss how EG-VEGF controls the two main types of placental angiogenesis, the intravilli and the fetomaternal interface one.

3.1. EG-VEGF Effects on Fetomaternal Angiogenesis. Trophoblastic invasion of spiral maternal arteries and decidua is the key process that establishes the fetomaternal circulation by the end of the first trimester of pregnancy. This process is known to be temporally and spatially controlled. Key studies from our group showed that EG-VEGF is a negative regulator of human EVT invasion. This statement was based on the demonstration that EG-VEGF inhibits EVT migration and invasion in HTR-8 cells (an extravillous trophoblastic cell line) and in first trimester villous explant culture systems and the demonstration that EG-VEGF inhibits HTR8 cells organization into tube-like structures [23]. These data strongly suggest that EG-VEGF acts as an inhibitor of trophoblast differentiation towards an invasive phenotype and are consistent with a model of normal placentation in which downregulation of EG-VEGF expression at around $11 \mathrm{wg}$ promotes differentiation of EVT. Therefore, the decrease in EG-VEGF circulating and placental levels at the end of the first trimester could contribute, with other factors, to extravillous trophoblast (EVT) invasion and to the establishment of fetomaternal circulation (Figure 1).

3.2. EG-VEGF Effects on Intravillous Angiogenesis. The placenta is composed of two types of endothelial cells: the microvascular endothelial cells (HPEC for human placental endothelial cells), cells that lie in the fetal capillaries of chorionic villi, and the umbilical vein macrovascular endothelial cells (HUVEC). It is well established that the endothelial cells that comprise the vascular beds of specific tissues are extremely diverse and display numerous tissue-specific characteristics in their phenotypes, growth properties, functions, and ultrastructure such as the intercellular junctions or the presence of fenestrae (for reviews see [30-32]). In accordance with these data, HPEC clearly differ from HUVEC in their phenotype and physiological functions [33-35]. HPEC show a spindle-shape that largely differs from the more polygonal phenotype of HUVEC [33-35]. HPEC grown in vitro secrete higher amounts of thromboxane and angiotensin II than HUVEC [34]. Furthermore, HPEC also show higher proliferative responses to tissue-restricted mediators (i.e., PlGF) in comparison to HUVEC [33-35]. Interestingly, ubiquitous angiogenic factors (i.e., FGF-2 and VEGF-A) exhibit similar effects on HPEC and HUVEC, suggesting that some tissuerestricted factors might contribute to endothelial singularity $[6,7,33-35]$.
In 2010, the angiogenic effects of EG-VEGF have been investigated in HPEC and HUVEC. Interestingly, EG-VEGF displayed specificity towards distinct vascular beds with major effects on HPEC-mediated angiogenesis (Figure 2). EG-VEGF increased HPEC proliferation, migration, tube-like formation, and sprouting, without affecting HUVEC-mediated angiogenesis. Both EG-VEGF receptors are expressed in vivo by placental HPEC and HUVEC. Quantification of PROKR1 and PROKR2 protein levels in endothelial cell primary cultures revealed larger expression of both receptors in HPEC than in HUVEC. This difference suggests a higher sensitivity of HPEC for EG-VEGF. Altogether, these data confirm the two distinct endothelial identities of HPEC and HUVEC and stress the importance to investigate placental angiogenesis with appropriate microvascular endothelial models.

The understanding of the mechanisms underlying placental angiogenesis was significantly improved by the use of in vitro models using appropriate endothelial cell cultures. In the last decades, numerous two- and three-dimensional assays helped to bring new insight into the understanding of EG-VEGF-mediated placental angiogenesis.

\subsubsection{Investigation of EG-VEGF Angiogenic Roles Using 2D-Primary Culture Models}

(i) Placental Endothelial Cells Primary Culture. HPEC can be successfully isolated from the placental microvasculature by enzymatic perfusion of the placenta $[21,34]$ or from digestion of placental tissues [36-40]. Despite growing evidence demonstrating placental endothelial heterogeneity, HUVEC are still the most commonly used cell type for angiogenesis studies $[8,41]$. Nevertheless, their above-mentioned differences in phenotype, gene expression, and physiology substantiate that microvascular endothelial cells are the unique model to use to investigate placental angiogenesis.

(ii) EG-VEGF Effect on HPEC Proliferation and Survival. Using complementary 2D-models, recent experiments have established the positive effect of EG-VEGF on HPEC proliferation and survival [21]. HPEC proliferation has been shown to be stimulated under EG-VEGF treatment, as assessed by $[3 \mathrm{H}]$-thymidine incorporation and $\mathrm{Ki}-67$ staining [21]. EG-VEGF was also shown to promote endothelial survival as evidenced by decreased caspase-3 activity [21]. These results demonstrate that EG-VEGF is a new mitogenic and prosurvival factor for microvascular endothelial cells of the placenta [21].

(iii) EG-VEGF Effect on Placental Endothelial Cell Migration and Tubulogenesis. Using a quantifiable 2D-model of tubulogenesis, we established that EG-VEGF strongly promotes HPEC morphogenesis into tube-like structures [42-44]. Using a monolayer wound-healing assay, EG-VEGF effect on HPEC migration has been investigated. This assay is one of the earliest developed methods to study directional cell migration in vitro [45]. Our data demonstrates that EG-VEGF 


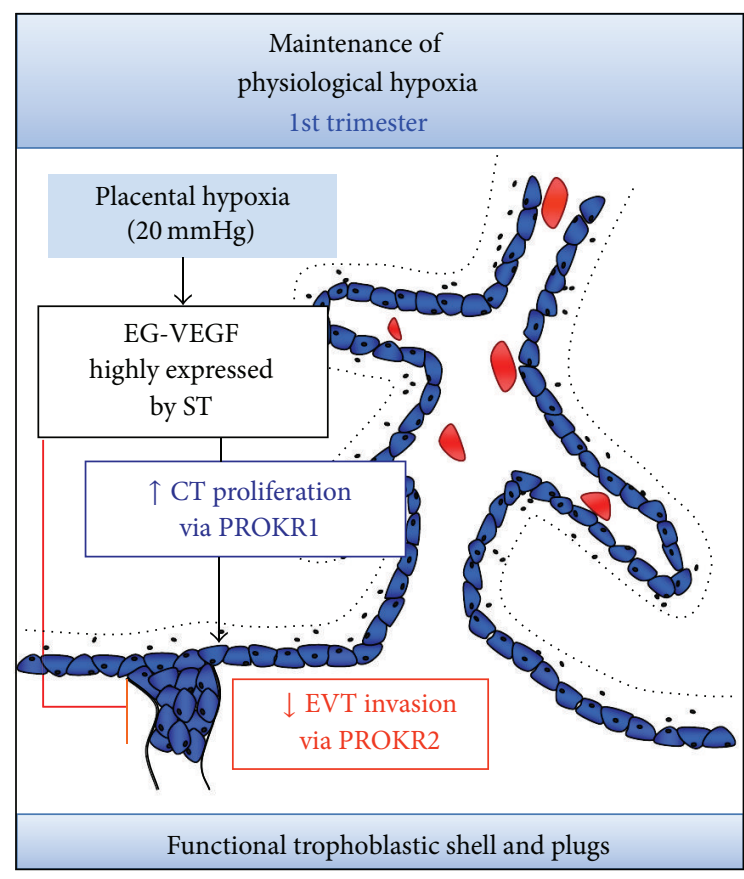

(a)

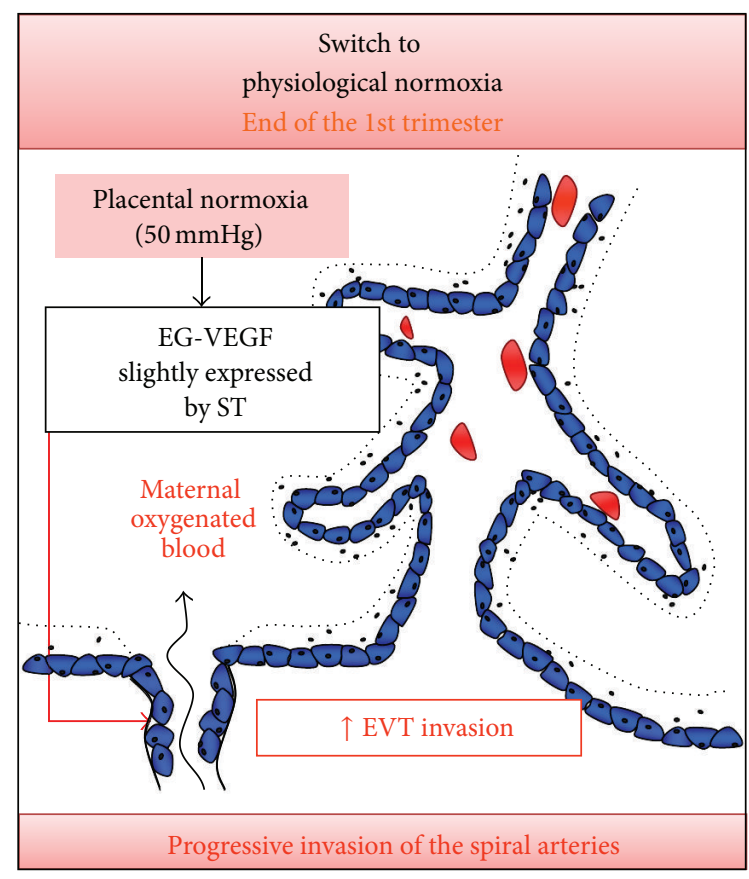

(b)

FIGURE 1: Proposed model of EG-VEGF-mediated effects on the fetomaternal angiogenesis during the first trimester of pregnancy. (a) and (b) represent cartoons of placental villi with EG-VEGF expression and actions on CT and EVT. (a) During the first trimester, EG-VEGF increases CT proliferation via PROKR1 activation and inhibits EVT invasion via PROKR2 activation. EG-VEGF/PROKR actions participate actively in trophoblastic shell and plugs constitution and contribute to the maintenance of physiological hypoxia during the first trimester of pregnancy. (b) At the end of the first trimester, EG-VEGF secretion declines. This contributes with other factors to EVT invasion and to the establishment of the fetomaternal circulation.

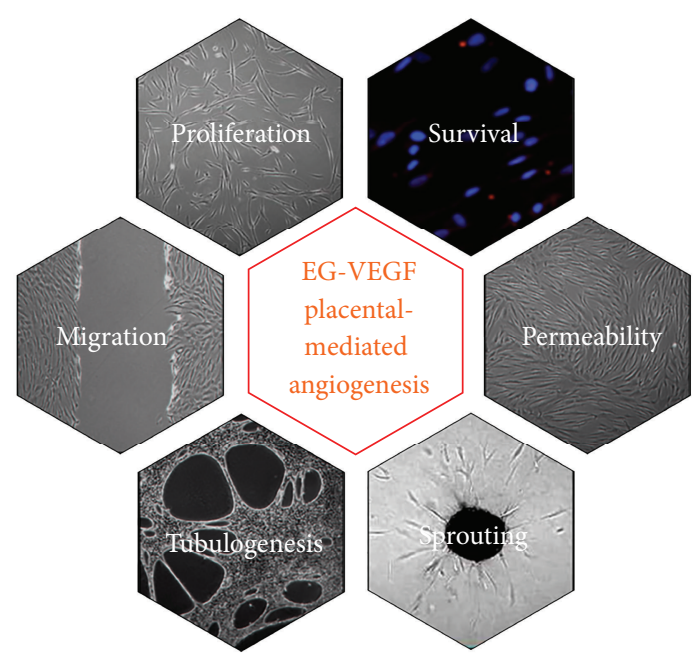

FIGURE 2: EG-VEGF is a new placental angiogenic factor. It controls placental growth via its multiple actions on endothelial cells within the chorionic villi.

significantly increased the migration of HPEC in this model [21].

(iv) EG-VEGF Effect on Placental Endothelial Permeability. In the placenta, the microvascular endothelium is known to form a selective permeable interface that participates in the fetomaternal transports of solutes and nutrients. Therefore, the maintenance of a semipermeable endothelium is critically important for the development of the fetus. Placental microvasculature is not static and can be modulated by exposure to specific stimuli that affect intracellular permeability and paracellular transport. Using the HPEC model, we demonstrated that EG-VEGF increased both transendothelial permeability and paracellular transport [21]. Measurement of the ion flux through the primary HPEC monolayer was evaluated by transendothelial electrical resistance (TEER) measurement [21]. The use of siRNAs and blocking antibodies demonstrated that PROKR2 was specifically mediating EG-VEGF effects on cell permeability. In addition, the effect of EG-VEGF on the paracellular transport was also investigated by measuring $[3 \mathrm{H}]$-mannitol transport through HPEC monolayer. We observed that EGVEGF almost doubled the $[3 \mathrm{H}]$-mannitol transport capacities of HPEC [21]. These results imply that EG-VEGF controls not only placental angiogenesis but also some physiological properties of placental vasculature such as permeability and transport of solute molecules. Altogether, the results suggest that EG-VEGF acts as a vascular bed-specific angiogenic factor providing an optimal vascular supply during human pregnancy.

3.2.2. Investigation of EG-VEGF Angiogenic Roles Using 3DPrimary Culture Models. In addition to two-dimensional cell 
culture systems, 3D-models have also been employed to investigate EG-VEGF effect on placental angiogenesis.

(i) EG-VEGF Effect on Intravilli Vascularization Using Placental Explant Model. Explants of human placenta are commonly used to study many tissue functions including cellular proliferation and differentiation [46]. In this system, EG-VEGF has been described to increase the number of differentiated endothelial cells (CD31+) within the villous tissue, suggesting an increase in the vascularisation within the placental villi [46]. This result is consistent with its proliferative and prosurvival effects observed in the $2 \mathrm{D}$-primary microvascular cells model [21].

(ii) EG-VEGF Effect on Endothelial Cell Sprouting. The 3D endothelial spheroid model was used to study the role of EG-VEGF on placental endothelial cell sprouting. EG-VEGF significantly increased HPEC sprouting in a dose-dependent manner. Importantly, EG-VEGF treatment has a stronger effect than VEGF on HPEC sprouting [21]. The use of 3Dendothelial spheroid models confirmed the positive effect of EG-VEGF on placental angiogenesis previously reported using 2D-models. The use of siRNAs and blocking antibodies demonstrated that the effect of EG-VEGF on HPEC sprouting was specifically mediated by PROKR1. Such a selectivity of PROKR1 action in angiogenesis has also been found in other organs [12-14].

Using multifaceted strategies that included molecular, immunochemical, and functional approaches, several recent publications have shed lights on EG-VEGF key roles in placental angiogenesis via its specific effect on microvascular endothelial cells proliferation, migration, survival, tube organization, sprouting, permeability, and paracellular transport. Altogether, these findings imply that EG-VEGF might act in concert with other angiogenic factors to coordinate series of events that ensure the success of placental vascular development.

3.3. EG-VEGF, a Mediator of Placental Angiogenesis. During the last decade, many angiogenic actors have been described as regulators of the EG-VEGF/PROKR system, suggesting that EG-VEGF regulation of placental angiogenesis could be of direct or indirect form.

3.3.1. Hypoxia: A Key Actor of Placental Angiogenesis and a Regulator of EG-VEGF. The human placenta develops in a low oxygen environment from the beginning of implantation to the end of the first trimester of pregnancy, due to proliferative trophoblast plugs within the maternal arteries that restrict blood flow into the intervillous space. This physiological hypoxia plays a key role in the modulation of the expression of several angiogenic factors [47, 48], including EG-VEGF. Numerous studies demonstrated that EG-VEGF is upregulated by hypoxia suggesting that this cytokine might mediate some of its angiogenic effects.

3.3.2. Human Chorionic Gonadotropin (hCG): A Pivotal Hormone in Placental Angiogenesis That Increases EG-VEGF/
PROKR System. Increasing evidence suggests that angiogenic effect of hCG on placental endothelial cells could be mediated by prior induction of EG-VEGF [49-51]. EG-VEGF and hCG are mainly secreted by the syncytiotrophoblast layer and exhibit similar patterns of expression with a peak around $8-10 \mathrm{wg}$. Recent findings demonstrate a new physiological regulation of EG-VEGF/PROKR system by hCG during the first trimester of pregnancy [51]. Using placental explants and primary trophoblast cultures, it has been established that hCG significantly increases EG-VEGF mRNA synthesis and protein secretion via the activation of the cAMP and protein kinase A signaling pathway [51]. HCG also induces mRNA and protein expression of PROKR1 and PROKR2 in first trimester human placenta [51]. These results reveal a new role for hCG in human placentation through its stimulation of the EG-VEGF/PROKR system and might explain the peak of expression of EG-VEGF and its receptors during the first trimester of pregnancy $(8-11 \mathrm{wg})$. Moreover, EGVEGF/PROKR regulation by hCG strongly suggests that some of the angiogenesis effects of hCG on placental villi might be mediated by EG-VEGF [49-51]. HCG is involved in many important functions in placental angiogenesis, including HPEC proliferation and sprouting $[49,50,52]$.

3.3.3. MAPK and PI3K/AKT: Key Signaling Pathways of EGVEGF Angiogenesis. The MAPK and PI3K/AKT signaling pathways are highly involved in angiogenesis [53-57]. They play an essential role in the formation of normal blood vessels during development via their direct effects on endothelial cell proliferation, survival, differentiation, migration, and angiogenesis and contribute indirectly to the induction of angiogenesis by increasing the expression of numerous angiogenic factors such as VEGF, nitric oxide, and angiopoietin [5357]. In HPEC, EG-VEGF induces a strong phosphorylation of MAPK and AKT proteins [21]. These data confirm the involvement of EG-VEGF in HPEC migration and survival and highly suggest its contribution in the induction of others angiogenic factors. Further investigations are required to determine the veracity of this hypothesis.

\subsubsection{IL-8: A Crucial Placental Angiogenic Factor Upregulated} by EG-VEGF. Using third trimester placental explant model, a recent study has demonstrated IL-8 induction by EG-VEGF, potentially via activation of PROKR1 [24]. IL-8 is an important placental angiogenic factor that promotes endothelial cell chemotaxis and proliferation [58]. This cytokine is expressed in the human placenta throughout pregnancy and facilitates vascular permeability $[59,60]$. Moreover, IL-8 is upregulated by HIF1 $\alpha$ and is increased in conditions characterized by pathological angiogenesis such as placental vascular insufficiency $[39,61]$ and preeclampsia [62].

Altogether, these results demonstrate that EG-VEGF can directly and/or indirectly control placental angiogenesis. Hence, deregulations in EG-VEGF and/or its receptors could well be associated to vascular-associated pathologies during pregnancy. 


\section{Role of EG-VEGF in Pregnancy-Related Pathologies}

It is well established that placental development depends on controlled growth, invasion, and differentiation of the trophoblast cells and on an adequate vascular development [63]. Hence, placental angiogenesis is highly linked to fetoplacental growth and fetomaternal exchanges. Abnormal angiogenesis has been associated with different pregnancyrelated pathologies such as ectopic pregnancy, recurrent pregnancy loss (RPL), gestational trophoblastic diseases (GTD), preeclampsia (PE), and fetal growth restriction (FGR). EGVEGF/PROKR expressions vary across normal pregnancy and in complicated pregnancies [21-23, 27, 51, 64, 65]. Recent studies have established correlations between abnormal EGVEGF expression and pregnancy-specific diseases, ranging from miscarriage to intrauterine growth restriction and preeclampsia. These results strongly suggest that EG-VEGF deregulation could be associated with adverse pregnancy outcomes.

4.1. EG-VEGF in Recurrent Pregnancy Loss (RPL). Recently, several publications demonstrated the involvement of EGVEGF and its receptors in the etiology of RPL [66-68]. This pathology is widely attributed to chromosomal aneuploidy in the conceptus and/or to a deregulation in the expression of uterine factors. In the last decade, histological and ultrasound studies illustrated a link between recurrent miscarriage and abnormal vascularization in the placental bed, suggesting that early disturbance in placental vascular development might contribute to the pathogenesis of miscarriages [6971]. In 2010, EG-VEGF receptor gene polymorphisms and haplotypes have been associated with RPL [67]. These data advocate that a deregulation in EG-VEGF-mediated signaling pathways could affect placental angiogenesis contributing to the pathogenesis of RPL. Further investigations are required to validate this hypothesis.

4.2. EG-VEGF in Gestational Trophoblastic Diseases (GTD). Recent data have shown that maternal circulating levels of EG-VEGF are increased in patients undergoing molar pregnancies, a severe form of the gestational trophoblastic disease (GTD) [72]. GTD includes a wide spectrum of pathologies ranging from partial/complete hydatidiform moles to gestational trophoblastic tumors. Increasing data report a poor placental vascularization during the first trimester of GTD [23, 72-74]. EG-VEGF controls numerous angiogenic processes during the first trimester of pregnancy [64] and it is significantly increased by hCG [51], a hormone that is also highly upregulated in GTD. Altogether, these results suggest that EG-VEGF increased circulating levels reported in GTD could be a consequence of hCG deregulation and propose that its angiogenic effects might contribute to the pathogenesis of GTD during the first trimester of pregnancy. Further investigations are ongoing to identify the participation of EG-VEGF in the development of gestational trophoblastic diseases and to investigate the potential use of this placental specific factor for early diagnosis and treatment of GTD.
4.3. Preeclampsia (PE). Recent publications report EG-VEGF and PROKR1 deregulations in PE and suggest their involvements in the development of this pregnancy-related pathology $[23,75]$. PE is a systemic syndrome that is characterized by hypertension and proteinuria that appears around 22 weeks of gestation. PE affects approximately 5-6\% of pregnancies worldwide accounting for nearly $18 \%$ of maternal deaths $[76,77]$. The etiology of PE remains largely unknown but increasing evidences suggest that it originates from abnormal placentation. In the fetomaternal unit, PE is marked by insufficient trophoblast invasion and poor maternal spiral artery remodeling [78]. Further investigations pointed out the potential involvement of angiogenic factors and their receptors in PE development and stressed their potential importance in the prediction of its occurrence [79]. For instance, increased expression of soluble Flt1 and soluble endoglin in the maternal circulation weeks before the onset of PE has been reported and suggested as predisposing factors of the disease [79]. Recently, several findings strongly suggest that deregulation of EG-VEGF expression in the placenta might be associated with the development of PE [21-23, 27, $51,64,65]$. In 2008, we reported a significant increase in EG-VEGF levels in the sera of third trimester PE patients as compared to age-matched controls [23]. More recently, a significant decrease in EG-VEGF mRNA expression has been reported in PE placentas [75], suggesting that local expression of EG-VEGF could be impaired at the transcriptional level in PE placentas. Determining the impact of these deregulations on placental development is difficult, as the relationship between maternal EG-VEGF circulating level and its local expression in the placenta remains unclear. EG-VEGF is also upregulated by hypoxia and hCG, two factors that are highly associated with the occurrence of PE [80-83]. The dynamic profile of EG-VEGF expression throughout pregnancy and its control of trophoblast invasion and placental angiogenesis strongly suggest that this cytokine contributes to the etiology of PE. Altogether, these results show that systemic and local EG-VEGF deregulation is associated with this pathology in the third trimester of pregnancy. However, we cannot conclude whether abnormal EG-VEGF levels are a cause or consequence of PE development. Further studies are needed to clarify whether EG-VEGF could be a predictive marker of PE.

4.4. Fetal Growth Restriction (FGR). Optimal growth of the fetus throughout pregnancy depends on an adequate vascular network in the fetomaternal unit [84]. Therefore, abnormalities in placental microvascular development toughly compromise the supply of nutrients and hormones, leading ultimately to fetal growth restriction [85]. Interestingly, recent findings reported that EG-VEGF circulating levels were significantly higher in FGR patients during the third trimester of pregnancy [27]. These results were confirmed at the placental level where significant increases in EG-VEGF, PROKR1, and PROKR2 mRNA and protein expression were also found [27]. The authors proposed two hypotheses that could explain the association between EG-VEGF/PROKR system upregulation 
and the FGR condition. The first one proposed that EGVEGF increased levels in FGR pregnancies could be a cause of the pathology, as sustained expression of EG-VEGF over the first trimester of pregnancy may compromise the spiral arteries remodeling and contribute to utero-placental hypoxia, a key parameter in the etiology of FGR [86, 87]. The second hypothesis proposed that FGR condition is caused by other predisposing factors that consequently increase EGVEGF/PROKR system in the placenta and sera of the patients. This hypothesis is supported by recent in vitro experiments that demonstrate the strong upregulation of EG-VEGF and its receptors by hypoxia and hCG $[22,51]$, two parameters that are known to be increased in FGR [86-89]. Further studies are required to determine whether EG-VEGF/PROKR deregulation is a cause or consequence of FGR.

Altogether, these results clearly demonstrate that EGVEGF and its receptors are closely associated to several pathologies marked by deregulated placental angiogenesis. These recent publications bring evidences for EG-VEGF association to key angiogenic processes and support the interest of new investigations on the predictive value of this factor in several pregnancy-associated pathologies including recurrent pregnancy loss, gestational trophoblastic disease, and placental pathologies associated with fetal growth restriction and/or preeclampsia.

\section{Concluding Remarks}

Disruption in the balance of placental angiogenesis controlling factors may lead to abnormal vascular development and compromises the success of pregnancy. Alterations in numerous specific angiogenic-signaling pathways have been already described in pregnancy-related diseases. The multiple roles of EG-VEGF in the development of the chorionic villi argue for its clinical relevance as a diagnostic and/or prognostic marker for several placental diseases. The current challenge in the field of reproduction is to discover early biomarkers of abnormal placental angiogenesis to develop successful screening tests for pregnancy disorders. These biomarkers also represent potential new therapeutic targets to "rescue" placental vascular development and thus fetal growth in compromised pregnancies. In the last decade, compelling advances highlighted the pivotal role of EG-VEGF and its receptors in regard to their expressions, multiple roles, and regulations in normal and pathological human pregnancies. These fundamental and clinical results highly suggest that EG-VEGF might be a potential early marker for several pathologies including recurrent pregnancy loss, gestational trophoblastic diseases, FGR, and PE. Further studies are required to evaluate its potential relevance as an early marker of these pregnancy-associated pathologies, probably in combination with other predictive parameters such as uterine arteries blood flow measurements by Doppler ultrasound imaging [90].

\section{Conflict of Interests}

The authors declare that there is no conflict of interests regarding the publication of this paper.

\section{References}

[1] G. J. Burton and E. Jauniaux, "Sonographic, stereological and Doppler flow velocimetric assessments of placental maturity," British Journal of Obstetrics and Gynaecology, vol. 102, no. 10, pp. 818-825, 1995.

[2] M. R. Jackson, T. M. Mayhew, and P. A. Boyd, "Quantitative description of the elaboration and maturation of villi from 10 weeks of gestation to term," Placenta, vol. 13, no. 4, pp. 357-370, 1992.

[3] D. S. Charnock-Jones, P. Kaufmann, and T. M. Mayhew, "Aspects of human fetoplacental vasculogenesis and angiogenesis. I. Molecular regulation," Placenta, vol. 25, no. 2-3, pp. 103113, 2004.

[4] P. Kaufmann, T. M. Mayhew, and D. S. Charnock-Jones, "Aspects of human fetoplacental vasculogenesis and angiogenesis. II. Changes during normal pregnancy," Placenta, vol. 25, no. 2-3, pp. 114-126, 2004.

[5] K. Benirschke and P. Kaufmann, Pathology of the Human Placenta, Springer, New Tork, NY, USA, 4th edition, 2000.

[6] M. Dellian, B. P. Witwer, H. A. Salehi, F. Yuan, and R. K. Jain, "Quantitation and physiological characterization of angiogenic vessels in mice: effect of basic fibroblast growth factor, vascular endothelial growth factor/vascular permeability factor, and host microenvironment," American Journal of Pathology, vol. 149, no. 1, pp. 59-71, 1996.

[7] G. E. Palade, M. Simionescu, and N. Simionescu, "Structural aspects of the permeability of the microvascular endothelium," Acta Physiologica Scandinavica, vol. 106, no. 463, pp. 11-32, 1979.

[8] W. G. Roberts, J. Delaat, M. Nagane, S. Huang, W. K. Cavenee, and G. E. Palade, "Host microvasculature influence on tumor vascular morphology and endothelial gene expression," The American Journal of Pathology, vol. 153, no. 4, pp. 1239-1248, 1998.

[9] P. A. Stewart and M. J. Wiley, "Developing nervous tissue induces formation of blood-brain barrier characteristics in invading endothelial cells: a study using quail-chick transplantation chimeras," Developmental Biology, vol. 84, no. 1, pp. 183$192,1981$.

[10] J. Lecouter, J. Kowalski, J. Foster et al., "Identification of an angiogenic mitogen selective for endocrine gland endothelium," Nature, vol. 412, no. 6850, pp. 877-884, 2001.

[11] D. C. Lin, C. M. Bullock, F. J. Ehlert, J. L. Chen, H. Tian, and Q. Y. Zhou, "Identification and molecular characterization of two closely related $G$ protein-coupled receptors activated by prokineticins/endocrine gland vascular endothelial growth factor," Journal of Biological Chemistry, vol. 277, no. 22, pp. 19276-19280, 2002.

[12] K. Urayama, D. B. Dedeoglu, C. Guilini et al., "Transgenic myocardial overexpression of prokineticin receptor-2 (GPR73b) induces hypertrophy and capillary vessel leakage," Cardiovascular Research, vol. 81, no. 1, pp. 28-37, 2009.

[13] K. Urayama, C. Guilini, N. Messaddeq et al., "The prokineticin receptor-1 (GPR73) promotes cardiomyocyte survival and angiogenesis," The FASEB Journal, vol. 21, no. 11, pp. 2980 2993, 2007.

[14] C. Guilini, K. Urayama, G. Turkeri et al., "Divergent roles of prokineticin receptors in the endothelial cells: angiogenesis and fenestration," The American Journal of Physiology-Heart and Circulatory Physiology, vol. 298, no. 3, pp. H844-H852, 2010.

[15] T. Kisliouk, N. Levy, A. Hurwitz, and R. Meidan, "Presence and regulation of endocrine gland vascular endothelial growth 
factor/prokineticin-1 and its receptors in ovarian cells," Journal of Clinical Endocrinology and Metabolism, vol. 88, no. 8, pp. 3700-3707, 2003.

[16] T. Kisliouk, H. Podlovni, and R. Meidan, "Unique expression and regulatory mechanisms of EG-VEGF/prokineticin-1 and its receptors in the corpus luteum," Annals of Anatomy, vol. 187, no. 5-6, pp. 529-537, 2005.

[17] R. D. Catalano, T. R. M. Lannagan, M. Gorowiec, F. C. Denison, J. E. Norman, and H. N. Jabbour, "Prokineticins: novel mediators of inflammatory and contractile pathways at parturition?" Molecular Human Reproduction, vol. 16, no. 5, pp. 311-319, 2010.

[18] T. Kisliouk, H. Podlovni, K. Spanel-Borowski, O. Ovadia, Q. Y. Zhou, and R. Meidan, "Prokineticins (endocrine gland-VEGF and BV8) in the bovine ovary: expression and role as mitogens and survival factors for corpus luteum derived-endothelial cells," Endocrinology, vol. 146, no. 9, pp. 3950-3958, 2005.

[19] J. LeCouter and N. Ferrara, "EG-VEGF and the concept of tissue-specific angiogenic growth factors," Seminars in Cell and Developmental Biology, vol. 13, no. 1, pp. 3-8, 2002.

[20] R. Lin, J. LeCouter, J. Kowalski, and N. Ferrara, "Characterization of endocrine gland-derived vascular endothelial growth factor signaling in adrenal cortex capillary endothelial cells," Journal of Biological Chemistry, vol. 277, no. 10, pp. 8724-8729, 2002.

[21] S. Brouillet, P. Hoffmann, M. Benharouga et al., "Molecular characterization of EG-VEGF-mediated angiogenesis: differential effects on microvascular and macrovascular endothelial cells," Molecular Biology of the Cell, vol. 21, no. 16, pp. 2832-2843, 2010.

[22] P. Hoffmann, J.-J. Feige, and N. Alfaidy, "Expression and oxygen regulation of endocrine gland-derived vascular endothelial growth factor/prokineticin-1 and its receptors in human placenta during early pregnancy," Endocrinology, vol. 147, no. 4, pp. 1675-1684, 2006.

[23] P. Hoffmann, Y. Saoudi, M. Benharouga et al., "Role of EGVEGF in human placentation: physiological and pathological implications," Journal of Cellular and Molecular Medicine, vol. 13, no. 8 B, pp. 2224-2235, 2009.

[24] F. C. Denison, S. Battersby, A. E. King, M. Szuber, and H. N. Jabbour, "Prokineticin-1: a novel mediator of the inflammatory response in third-trimester human placenta," Endocrinology, vol. 149, no. 7, pp. 3470-3477, 2008.

[25] M. Matjila, R. Millar, Z. van der Spuy, and A. Katz, "The differential expression of Kiss1, MMP9 and angiogenic regulators across the feto-maternal interface of healthy human pregnancies: implications for trophoblast invasion and vessel development," PLoS ONE, vol. 8, no. 5, Article ID e63574, 2013.

[26] J. Evans, R. D. Catalano, K. Morgan, H. O. D. Critchley, R. P. Millar, and H. N. Jabbour, "Prokineticin 1 signaling and gene regulation in early human pregnancy," Endocrinology, vol. 149, no. 6, pp. 2877-2887, 2008.

[27] S. Brouillet, P. Murthi, P. Hoffmann et al., "EG-VEGF controls placental growth and survival in normal and pathological pregnancies: case of fetal growth restriction (FGR)," Cellular and Molecular Life Sciences, vol. 70, no. 3, pp. 511-525, 2013.

[28] E. Jauniaux, J. Hempstock, N. Greenwold, and G. J. Burton, "Trophoblastic oxidative stress in relation to temporal and regional differences in maternal placental blood flow in normal and abnormal early pregnancies," The American Journal of Pathology, vol. 162, no. 1, pp. 115-125, 2003.
[29] G. J. Burton and E. Jauniaux, "Placental oxidative stress: from miscarriage to preeclampsia," Journal of the Society for Gynecologic Investigation, vol. 11, no. 6, pp. 342-352, 2004.

[30] B. R. Zetter, "The endothelial cells of large and small blood vessels," Diabetes, vol. 30, no. 2, pp. 24-28, 1981.

[31] L. F. Fajardo, "Special report. The complexity of endothelial cells. A review," The American Journal of Clinical Pathology, vol. 92, no. 2, pp. 241-250, 1989.

[32] R. Bicknell, "Heterogeneity of the endothelial cell," Behring Institute Mitteilungen, no. 92, pp. 1-7, 1993.

[33] I. Lang, C. Hoffmann, H. Olip et al., "Differential mitogenic responses of human macrovascular and microvascular endothelial cells to cytokines underline their phenotypic heterogeneity," Cell Proliferation, vol. 34, no. 3, pp. 143-155, 2001.

[34] I. Lang, M. A. Pabst, U. Hiden et al., "Heterogeneity of microvascular endothelial cells isolated from human term placenta and macrovascular umbilical vein endothelial cells," European Journal of Cell Biology, vol. 82, no. 4, pp. 163-173, 2003.

[35] I. Lang, M. Hartmann, A. Blaschitz, G. Dohr, G. Skofitsch, and G. Desoye, "Immunohistochemical evidence for the heterogeneity of maternal and fetal vascular endothelial cells in human full-term placenta," Cell and Tissue Research, vol. 274, no. 2, pp. 211-218, 1993.

[36] J. C. Challier, A. Kacemi, and G. Olive, "Mixed culture of pericytes and endothelial cells from fetal microvessels of the human placenta," Cellular and Molecular Biology, vol. 41, no. 2, pp. 233-241, 1995.

[37] A. Kacemi, M. Galtier, M. J. Espié, and J. C. Challier, "Isolation of villous microvessels from the human placenta," Comptes Rendus de l'Académie des Sciences III, vol. 320, no. 2, pp. 171-177, 1997.

[38] B. Ugele and F. Lange, "Isolation of endothelial cells from human placental microvessels: effect of different proteolytic enzymes on releasing endothelial cells from villous tissue," In Vitro Cellular \& Developmental Biology, vol. 37, no. 7, pp. 408413, 2001.

[39] X. Wang, N. Athayde, and B. Trudinger, "A proinflammatory cytokine response is present in the fetal placental vasculature in placental insufficiency," The American Journal of Obstetrics and Gynecology, vol. 189, no. 5, pp. 1445-1451, 2003.

[40] J. F. Dye, S. Vause, T. Johnston et al., "Characterization of cationic amino acid transporters and expression of endothelial nitric oxide synthase in human placental microvascular endothelial cells," The FASEB Journal, vol. 18, no. 1, pp. 125-127, 2004.

[41] P. Murthi, M. So, N. M. Gude, V. L. Doherty, S. P. Brennecke, and B. Kalionis, "Homeobox genes are differentially expressed in macrovascular human umbilical vein endothelial cells and microvascular placental endothelial cells," Placenta, vol. 28, no. 2-3, pp. 219-223, 2007.

[42] J. D. Murray, "On the mechanochemical theory of biological pattern formation with application to vasculogenesis," Comptes Rendus-Biologies, vol. 326, no. 2, pp. 239-252, 2003.

[43] G. E. Davis and D. R. Senger, "Endothelial extracellular matrix: biosynthesis, remodeling, and functions during vascular morphogenesis and neovessel stabilization," Circulation Research, vol. 97, no. 11, pp. 1093-1107, 2005.

[44] D. Donovan, N. J. Brown, E. T. Bishop, and C. E. Lewis, "Comparison of three in vitro human "angiogenesis" assays with capillaries formed in vivo," Angiogenesis, vol. 4, no. 2, pp. 113$121,2001$. 
[45] L. G. Rodriguez, X. Wu, and J. L. Guan, "Wound-healing assay," Methods in Molecular Biology, vol. 294, pp. 23-29, 2005.

[46] R. K. Miller, O. Genbacev, M. A. Turner, J. D. Aplin, I. Caniggia, and B. Huppertz, "Human placental explants in culture: approaches and assessments," Placenta, vol. 26, no. 6, pp. 439448, 2005.

[47] O. Genbacev, Y. Zhou, J. W. Ludlow, and S. J. Fisher, "Regulation of human placental development by oxygen tension," Science, vol. 277, no. 5332, pp. 1669-1672, 1997.

[48] G. J. Burton, E. Jauniaux, and A. L. Watson, "Maternal arterial connections to the placental intervillous space during the first trimester of human pregnancy: the Boyd Collection revisited," The American Journal of Obstetrics and Gynecology, vol. 181, no. 3, pp. 718-724, 1999.

[49] M. Zygmunt, F. Herr, S. Keller-Schoenwetter et al., "Characterization of human chorionic gonadotropin as a novel angiogenic factor," Journal of Clinical Endocrinology and Metabolism, vol. 87, no. 11, pp. 5290-5296, 2002.

[50] D. Islami, P. Bischof, and D. Chardonnens, "Modulation of placental vascular endothelial growth factor by leptin and hCG," Molecular Human Reproduction, vol. 9, no. 7-8, pp. 395-398, 2003.

[51] S. Brouillet, P. Hoffmann, S. Chauvet et al., "Revisiting the role of hCG: new regulation of the angiogenic factor EG-VEGF and its receptors," Cellular and Molecular Life Sciences, vol. 69, no. 9, pp. 1537-1550, 2012.

[52] F. Herr, N. Baal, K. Reisinger et al., "HCG in the regulation of placental angiogenesis. Results of an in vitro study," Placenta, vol. 28, supplement 1, pp. S85-S93, 2007.

[53] G. Sa, G. Murugesan, M. Jaye, Y. Ivashchenko, and P. L. Fox, "Activation of cytosolic phospholipase A2 by basic fibroblast growth factor via a p42 mitogen-activated protein kinasedependent phosphorylation pathway in endothelial cells," Journal of Biological Chemistry, vol. 270, no. 5, pp. 2360-2366, 1995.

[54] T. Matsumoto, I. Turesson, M. Book, P. Gerwins, and L. Claesson-Welsh, "p38 MAP kinase negatively regulates endothelial cell survival, proliferation, and differentiation in FGF-2-stimulated angiogenesis," Journal of Cell Biology, vol. 156, no. 1, pp. 149-160, 2002.

[55] K. Kuida and D. M. Boucher, "Functions of MAP kinases: Insights from gene-targeting studies," Journal of Biochemistry, vol. 135, no. 6, pp. 653-656, 2004.

[56] J. Karar and A. Maity, "PI3K/AKT/mTOR Pathway in Angiogenesis," Frontiers in Molecular Neuroscience, vol. 4, article 51, 2011.

[57] K. Wang and J. Zheng, "Signaling regulation of fetoplacental angiogenesis," Journal of Endocrinology, vol. 212, no. 3, pp. 243255, 2012.

[58] U. A. Kayisli, R. Demir, G. Erguler, and A. Arici, "Vasodilatorstimulated phosphoprotein expression and its cytokinemediated regulation in vasculogenesis during human placental development," Molecular Human Reproduction, vol. 8, no. 11, pp. 1023-1030, 2002.

[59] S. Saito, T. Kasahara, S. Sakakura, H. Umekage, N. Harada, and M. Ichijo, "Detection and localization of interleukin-8 mRNA and protein in human placenta and decidual tissues," Journal of Reproductive Immunology, vol. 27, no. 3, pp. 161-172, 1994.

[60] I. G. Colditz, "Effect of exogenous prostaglandin E2 and actinomycin D on plasma leakage induced by neutrophil-activating peptide-1/interleukin-8," Immunology and Cell Biology, vol. 68, no. 6 , pp. 397-403, 1990.
[61] K. S. Kim, V. Rajagopal, C. Gonsalves, C. Johnson, and V. K. Kalra, "A novel role of hypoxia-inducible factor in cobalt chloride- and hypoxia-mediated expression of IL-8 chemokine in human endothelial cells," Journal of Immunology, vol. 177, no. 10, pp. 7211-7224, 2006.

[62] R. S. Bowen, Y. Gu, Y. Zhang, D. F. Lewis, and Y. Wang, "Hypoxia promotes interleukin- 6 and -8 but reduces interleukin-10 production by placental trophoblast cells from preeclamptic pregnancies," Journal of the Society for Gynecologic Investigation, vol. 12, no. 6, pp. 428-432, 2005.

[63] M. J. Cross, J. Dixelius, T. Matsumoto, and L. Claesson-Welsh, "VEGF-receptor signal transduction," Trends in Biochemical Sciences, vol. 28, no. 9, pp. 488-494, 2003.

[64] S. Brouillet, P. Hoffmann, J. J. Feige, and N. Alfaidy, "EG-VEGF: a key endocrine factor in placental development," Trends in Endocrinology and Metabolism, vol. 23, no. 10, pp. 501-508, 2012.

[65] S. Brouillet, P. Hoffmann, C. Thomas-Cadi et al., "PROK1, prognostic marker of embryo implantation?" Gynécologie Obstétrique \& Fertilité, vol. 41, no. 9, pp. 562-565, 2013.

[66] M. T. Su, S. H. L., Y. C. Chen, L. W. Wu, and P. L. Kuo, "Prokineticin receptor variants (PKR1-I379V and PKR2-V331M) are protective genotypes in human early pregnancy," Reproduction, vol. 146, no. 1, pp. 63-73, 2013.

[67] M. T. Su, S. H. Lin, I. W. Lee et al., "Polymorphisms of endocrine gland-derived vascular endothelial growth factor gene and its receptor genes are associated with recurrent pregnancy loss," Human Reproduction, vol. 25, no. 11, pp. 2923-2930, 2010.

[68] M. Salker, G. Teklenburg, M. Molokhia et al., "Natural selection of human embryos: impaired decidualization of endometrium disables embryo-maternal interactions and causes recurrent pregnancy loss," PLoS ONE, vol. 5, no. 4, Article ID e10287, 2010.

[69] P. Vuorela, O. Carpén, M. Tulppala, and E. Halmesmäki, "VEGF, its receptors and the Tie receptors in recurrent miscarriage," Molecular Human Reproduction, vol. 6, no. 3, pp. 276-282, 2000.

[70] M. Zygmunt, F. Herr, K. Münstedt, U. Lang, and O. D. Liang, "Angiogenesis and vasculogenesis in pregnancy," European Journal of Obstetrics \& Gynecology and Reproductive Biology, vol. 110, supplement 1, pp. S10-S18, 2003.

[71] M. M. Manolea, O. A. Gavrilă, F. C. Popescu, L. Novac, and G. O. Mateescu, "The importance of immunohistochemical evaluation of the vascular changes from the decidua and placenta in recurrent pregnancy loss," Romanian Journal of Morphology and Embryology, vol. 53, no. 2, pp. 363-368, 2012.

[72] H. Boufettal, J. J. Feige, M. Benharouga et al., "Potential role of the angiogenic factor, "EG-VEGF" in gestational trophoblastic diseases," Pathologie Biologie, vol. 61, no. 5, pp. 178-183, 2013.

[73] F. Bolat, N. Haberal, N. Tunali, E. Aslan, N. Bal, and I. Tuncer, "Expression of vascular endothelial growth factor (VEGF), hypoxia inducible factor 1 alpha (HIF-1 $\alpha$ ), and transforming growth factors $\beta 1$ (TGF $\beta 1)$ and $\beta 3$ (TGF $\beta 3)$ in gestational trophoblastic disease," Pathology Research and Practice, vol. 206, no. 1, pp. 19-23, 2010.

[74] Z. S. Tuncer, G. L. Vegh, V. Fulop, D. R. Genest, S. C. Mok, and R. S. Berkowitz, "Expression of epidermal growth factor receptorrelated family products in gestational trophoblastic diseases and normal placenta and its relationship with development of postmolar tumor," Gynecologic Oncology, vol. 77, no. 3, pp. 389393, 2000.

[75] M. Szuber, W. Markwitz, M. Ropacka, and G. H. Breborowicz, "The possible role of the PK1 and its receptor in the etiology of the preeclampsia," Neuroendocrinology Letters, vol. 32, no. 4, pp. 563-572, 2011. 
[76] WHO, World Health Report: Make Every Mother and Child Count, World Health Organization, Geneva, Switzerland, 2005.

[77] B. Sibai, G. Dekker, and M. Kupferminc, "Pre-eclampsia," The Lancet, vol. 365, no. 9461, pp. 785-799, 2005.

[78] J. W. Meekins, R. Pijnenborg, M. Hanssens, I. R. McFadyen, and A. Van Asshe, "A study of placental bed spiral arteries and trophoblast invasion in normal and severe pre-eclamptic pregnancies," British Journal of Obstetrics and Gynaecology, vol. 101, no. 8, pp. 669-674, 1994.

[79] R. J. Levine, S. E. Maynard, C. Qian et al., "Circulating angiogenic factors and the risk of preeclampsia," The New England Journal of Medicine, vol. 350, no. 7, pp. 672-683, 2004.

[80] L. A. Cole, "New discoveries on the biology and detection of human chorionic gonadotropin," Reproductive Biology and Endocrinology, vol. 7, article 8, 2009.

[81] D. F. Liu, L. H. Dickerman, and R. W. Redline, "Pathologic findings in pregnancies with unexplained increases in midtrimester maternal serum human chorionic gonadotropin levels," The American Journal of Clinical Pathology, vol. 111, no. 2, pp. 209215, 1999.

[82] R. Douglas Wilson, A. Gagnon et al., "Obstetrical complications associated with abnormal maternal serum markers analytes," Journal of Obstetrics \& Gynaecology, vol. 30, no. 10, pp. 918-949, 2008.

[83] L. A. Cole, "hCG and hyperglycosylated hCG in the establishment and evolution of hemochorial placentation," Journal of reproductive immunology, vol. 82, no. 2, pp. 112-118, 2009.

[84] J. Kingdom, B. Huppertz, G. Seaward, and P. Kaufmann, "Development of the placental villous tree and its consequences for fetal growth," European Journal of Obstetrics Gynecology and Reproductive Biology, vol. 92, no. 1, pp. 35-43, 2000.

[85] I. Cetin, J. M. Foidart, M. Miozzo et al., "Fetal growth restriction: a workshop report," Placenta, vol. 25, no. 8-9, pp. 753-757, 2004.

[86] T. Y. Khong, F. De Wolf, W. B. Robertson, and I. Brosens, "Inadequate maternal vascular response to placentation in pregnancies complicated by pre-eclampsia and by small-for-gestational age infants," British Journal of Obstetrics and Gynaecology, vol. 93, no. 10, pp. 1049-1059, 1986.

[87] Y. Zhou, C. H. Damsky, and S. J. Fisher, "Preeclampsia is associated with failure of human cytotrophoblasts to mimic a vascular adhesion phenotype: one cause of defective endovascular invasion in this syndrome?" Journal of Clinical Investigation, vol. 99, no. 9, pp. 2152-2164, 1997.

[88] B. Fitzgerald, K. Levytska, J. Kingdom, M. Walker, D. Baczyk, and S. Keating, "Villous trophoblast abnormalities in extremely preterm deliveries with elevated second trimester maternal serum hCG or inhibin-A," Placenta, vol. 32, no. 4, pp. 339-345, 2011.

[89] D. Krantz, L. Goetzl, J. L. Simpson et al., "Association of extreme first-trimester free human chorionic gonadotropin- $\beta$, pregnancy-associated plasma protein $A$, and nuchal translucency with intrauterine growth restriction and other adverse pregnancy outcomes," The American Journal of Obstetrics and Gynecology, vol. 191, no. 4, pp. 1452-1458, 2004.

[90] T. Todros, A. Sciarrone, E. Piccoli, C. Guiot, P. Kaufmann, and J. Kingdom, "Umbilical doppler waveforms and placental villous angiogenesis in pregnancies complicated by fetal growth restriction," Obstetrics and Gynecology, vol. 93, no. 4, pp. 499503,1999 . 

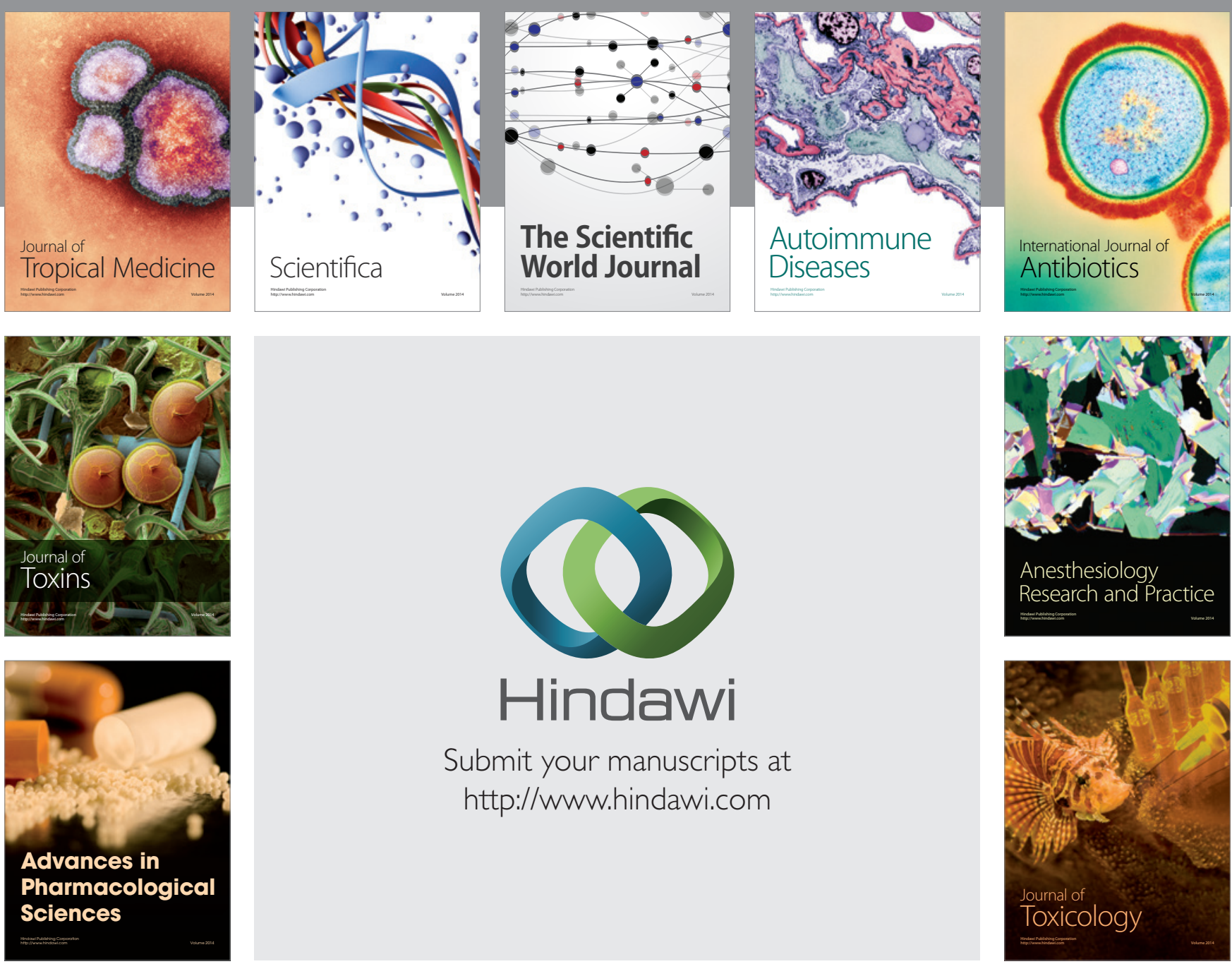

\section{Hindawi}

Submit your manuscripts at

http://www.hindawi.com
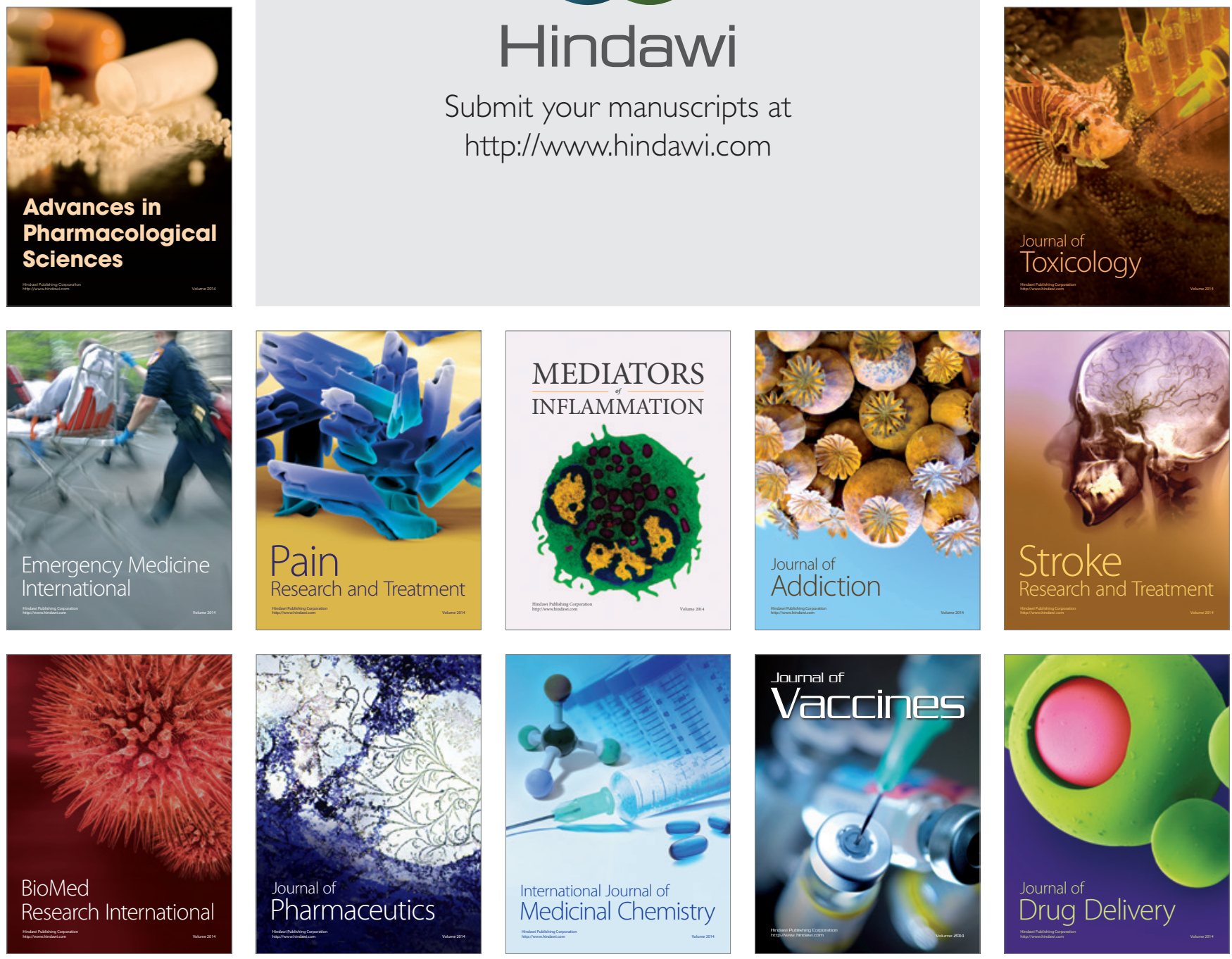